\title{
Marcas contrarias a las buenas costumbres y libertad de expresión
}

\section{Sentencia del Tribunal de Justicia de 27 de febrero de 2020 (C-248/18 P)}

\author{
$* * * *$ \\ Jesús Gómez Montero \\ Universidad Complutense de Madrid. Fundación Alberto Elzaburu \\ susogmontero@hotmail.com
}

Marca solicitada: FACK JU GÖTHE.

Norma jurídica aplicada: Artículo 7.1 f) Reglamento de la Marca de la Unión Europea (RMUE).

El análisis para comprobar si una marca es contraria a las buenas costumbres debe realizarse teniendo en cuenta la percepción que el público tiene de ella, valorando todas las circunstancias, incluida -cuando coincide con el nombre de una película- la referente a la aceptación social de su título y la valoración que de este haga el público pertinente.

La libertad de expresión, en cuanto derecho fundamental que debe garantizarse, ha de ser tenida en cuenta a la hora de valorar si una marca es contraria o no a las buenas costumbres.

\section{Tramitación administrativa ante la EUIPO}

CONSTANTIN FILM PRODUKTION GmbH solicitó el 21 abril de 2015 en la Oficina de la Propiedad Intelectual de la Unión Europea (EUIPO) la marca FACK JU GÖTHE para distinguir productos y servicios correspondientes a las clases 3, 9, 14, 16, 18, 21, 25, 28, 30, 32, 33, 38 y 41. Debe tenerse en cuenta, además, que esta denominación se corresponde con el título de una comedia cinematográfica alemana de gran éxito en 2013, con otras versiones también exitosas en 2015 (Fack ju Göthe 2) y 2017 (Fack ju Göthe 3).

La EUIPO denegó la marca mediante resolución de 25 de septiembre de 
2015 al entender el examinador que la primera parte de la denominación fack $j u$ (en alemán) se corresponde, a la hora de pronunciarse, con la expresión inglesa fuck you (traducida al español como "jódete"). Por ello, resultaba contraria al orden público y buenas costumbres y era de aplicación el artículo $7.1 \mathrm{f}$ ) del Reglamento 207/2009 sobre la Marca de la Unión Europea (RMUE), cuya finalidad era no permitir el registro de marcas vulgares, desagradables, insultantes, ofensivas, amenazantes.

La decisión del examinador fue refrendada por la Sala de Recursos de la EUIPO mediante resolución de 1 de diciembre de 2016.

\section{La sentencia del Tribunal General}

El recurso interpuesto por CONSTANTIN FILM ante el Tribunal General (TG) fue desestimado por la sentencia de 24 de enero de 2018 (T-69/17). ${ }^{1}$ Los argumentos utilizados para desestimar el recurso pueden sintetizarse de la siguiente manera:

a) La expresión fuck you (pronunciación en inglés de fuck ju) tiene un carácter vulgar y ofensivo, incluso aunque no sea entendida en su connotación sexual (existen otras acepciones como "enfado", "desconfianza", "desprecio hacia otra persona").

b) La marca está compuesta por un término - kuck ju- que es percibido por el público pertinente (alemán y austríaco) como intrínsecamente vulgar y ofensivo tratándose de una marca manifiestamente obscena y contraria a las buenas costumbres.

c) La inclusión del nombre Göthe (alusiva al nombre del respetado escritor Johan Wolfgang Goethe) no atenúa el carácter vulgar de la expresión fuck ju, sino que agrava la situación, pues implica un insulto póstumo al escritor de manera degradante y vulgar.

d) La circunstancia relativa a la fama del film no tiene incidencia favorable, ya que el público no percibe necesariamente las marcas y los títulos de las películas de la misma manera: (i) la finalidad del derecho de marcas es garantizar una competencia no falseada y no la libertad de expresión; (ii) el título de una película sirve para distinguir una obra artística de otra y para designar su contenido, actuando al mismo tiempo como una manifestación de la libertad de expresión y de la libertad artística.

1 Véase: http://curia.europa.eu/juris/document/document.jsf?text=\&docid=198722\&pageIndex$=0 \&$ doclang=FR\&mode $=1$ st $\&$ dir $=\&$ occ $=$ first $\&$ part $=1 \&$ cid $=5806366$. 


\section{La sentencia del Tribunal de Justicia}

Interpuesto el oportuno recurso de casación, el Tribunal de Justicia (TJ), en su sentencia de 27 de febrero de 2020 (C-240/18 P), ${ }^{2}$ lo estima y anula las decisiones del TG y de la Sala de Recursos. Los fundamentos que utiliza el TJ parten de unos supuestos completamente diferentes a los señalados por el TG y, en consecuencia, sus conclusiones también divergen. Concretamente, son los siguientes:

a) Se centra en el análisis de las buenas costumbres (excluye el orden público) y señala que no es equivalente al buen gusto, no bastando que una denominación resulte de mal gusto para denegar una marca.

b) Las buenas costumbres deben referenciarse a valores y normas morales fundamentales que pueden evolucionar a lo largo del tiempo y variar en el espacio, y deben determinarse con arreglo al consenso social predominante en cada sociedad en el momento de la evaluación. A efectos de dicha apreciación, se debe tener en cuenta el contexto social, incluidas, en su caso, las características culturales, religiosas o filosóficas que le son propias, con el fin de evaluar, de manera objetiva, lo que la mencionada sociedad considera moralmente aceptable en ese momento. c) La percepción de una marca como contraria a las "buenas costumbres" no debe realizarse en abstracto y requiere un análisis de todos los elementos propios del caso, con el fin de determinar de qué modo el público pertinente (una persona razonable con una sensibilidad y tolerancia medias) percibe un signo de este tipo.

El TJ parte de estos principios y critica las decisiones de los órganos inferiores, afirmando que realizaron una aproximación abstracta y teórica a la hora de analizar la denominación FACK JU GÖTHE sin tener en cuenta elementos concretos. Así, el TJ indica, en primer término, que el título de las comedias en cuestión y, por tanto, la marca solicitada no consisten en esta expresión inglesa como tal (fuck you), sino en una transcripción fonética distinta de esta al alemán (fuck ju), acompañada del elemento "Göhte".

Pero el argumento principal del TJ para estimar el recurso se basa en que el TG no podía limitarse a una mera apreciación abstracta de dicha marca y de la expresión inglesa a la que ese público asocia la primera parte de ella y, por ejemplo, tendría que haber valorado la circunstancia de que su título no parecía haber suscitado especial controversia entre el público de habla alemana.

El TJ desarrolla este argumento y manifiesta que el signo denominativo "Fack

2 Véase: http://curia.europa.eu/juris/document/document.jsf?text=\&docid=223843\&pageIndex$=0 \&$ doclang $=$ es $\&$ mode $=1$ st $\&$ dir $=\&$ occ $=$ first $\&$ part $=1 \&$ cid $=5805328$. 
Ju Göhte" corresponde al título de una comedia cinematográfica alemana, producida por la recurrente, que fue uno de los mayores éxitos cinematográficos del año 2013 en Alemania y que fue vista por varios millones de personas cuando se estrenó. Y el TJ termina afirmando que

si bien el éxito de una película no permite demostrar automáticamente la aceptación social de su título y de un signo denominativo epónimo, no es menos cierto que se trata, como mínimo, de un indicio de tal aceptación, que debe apreciarse a la luz de todos los elementos pertinentes del caso para establecer, de manera concreta, la percepción de ese signo cuando es usado como marca.

Todas estas circunstancias llevan al TJ a entender que el TG no había fundamentado correcta y suficientemente la denegación de la marca FUCK JU GÖHTE de tal manera que anule las resoluciones denegatorias de esta. Y para completar sus argumentos destaca, finalmente, el papel relevante que debe otorgarse al principio de la libertad de expresión en el derecho de marcas. A tal efecto señala:

Por último, cabe añadir que, contrariamente a lo que señala el Tribunal General en el apartado 29 de la sentencia recurrida, a tenor del cual "existe, en el ámbito del arte, de la cultura y de la literatura, una preocupación constante por preservar la libertad de expresión que no existe en el ámbito de las marcas", la libertad de expresión, que se recoge en el artículo 11 de la Carta de los Derechos Fundamentales de la Unión Europea, ha de ser [...] tenida en cuenta en la aplicación del artículo 7, apartado 1, letra f), del Reglamento n. ${ }^{\circ}$ 207/2009. Esta conclusión es corroborada además tanto por el considerando 21 del Reglamento 2015/2424, que modificó el Reglamento n. ${ }^{\circ}$ 207/2009, como por el considerando 21 del Reglamento 2017/1001, los cuales inciden expresamente en la necesidad de aplicar esos Reglamentos de manera que se garantice el pleno respeto de las libertades y de los derechos fundamentales, en particular de la libertad de expresión” ( apartado 56).

\section{Comentario}

No cabe duda de que la sentencia recaída en el caso Fuck ju Göhte puede tener una influencia importante en las resoluciones que en el futuro se tomen a la hora de aplicar el artículo 7.1. f) RMUE y, en consecuencia, en el análisis que debe realizarse de las marcas que puedan ser contrarias a las buenas costumbres (y, por extensión, al orden público). En este sentido, la sentencia comentada puede incidir en los tres aspectos básicos siguientes. 
En primer lugar, de la sentencia comentada se infiere que, para analizar esta prohibición, ya no resulta axiomático fijarse únicamente en el carácter intrínseco del significado que puede tener el signo que constituye la marca. En este sentido, debe recordarse que, en determinadas ocasiones, los tribunales habían manifestado que hay casos en los que el carácter ofensivo, soez etc. de una palabra era intrínseco a esta sin necesidad de otras valoraciones. Por el contrario, de la sentencia comentada se desprende que resultará siempre necesario que tal signo se analice en función de los elementos o circunstancias que existan en el momento de solicitarse la marca.

En segundo término, podría deducirse por omisión que el TJ no da una importancia decisiva a los productos o servicios que distinga la marca a la hora de evaluar si esta es contraria o no a las buenas costumbres; ello podemos deducirlo del hecho de que la sentencia comentada no hace ningún análisis concreto y diferenciado en relación con los productos o servicios distinguidos por la marca FUCK JU GÖHTE, que, recordemos, se aplica a una variadísima gama, pues la marca se solicitó para muchas clases del nomenclátor internacional.

Esta omisión, en mi opinión, es criticable, pues la naturaleza de los productos y la percepción del público a quien específicamente se dirigen o alcanzan es una circunstancia de verdadero valor para conocer si una marca es contraria, en cada caso concreto, a las buenas costumbres

Y, en tercer lugar, y este es el aspecto más interesante, el TJ se alinea con las tesis -imperantes ya en otras jurisdicciones, como la de Estados Unidos- que mantienen que, a la hora de analizar la prohibición de registrar marcas por ser contrarias a las buenas costumbres, deberá tenerse en cuenta el principio de la libertad de expresión.

Ciñéndonos a la Unión Europea, hay que partir de la base de que el principio de libertad de expresión es aplicable a las actividades empresariales y comerciales según ha establecido el Tribunal Europeo de Derechos Humanos. Asimismo, este Alto Tribunal Europeo mantiene desde hace décadas que la libertad de expresión ampara a la actividad publicitaria. Por ello, no debe extrańar que la tendencia actual vaya en la dirección de aceptar esa mayor injerencia del principio de la libertad de expresión en la actividad comercial.

Esta tendencia se aprecia también en el derecho de marcas y, particularmente, se inicia con la inclusión en el Considerando 21 de la Directiva 2015/2436 de Marcas de 16 de diciembre de 2015 y el Considerando 21 de RMUE 2017/1001 de 14 de julio de 2017, que incluyen a la libertad de expresión como una limitación impuesta a los titulares de marcas por el uso que terceros puedan realizar de esta con fines literarios, artísticos, de parodia, etcétera. 
El TJ hace una mención a tal circunstancia en la sentencia comentada al indicar que el Considerando 21 del RMUE reconoce tal principio. En mi opinión, el supuesto del Considerando 21 sirve para garantizar la libertad de expresión en el contexto de la utilización de la marca en una obra artística, intelectual, de crítica etc. y hay que ponerla en relación con el artículo 14 RMUE (limitación de los efectos de una marca de la Unión) y no con el artículo 7.1. f) RMUE. En todo caso, lo realmente importante es que el TJ ha reconocido claramente la inclusión del principio de la libertad de expresión en el Derecho de Marcas y, particularmente, en relación con la prohibicion de registro de marcas contrarias a las buenas costumbres.

De lo que no cabe duda es de que la evolución resulta imparable y, frente a la reiterada posición negatoria mantenida hasta este momento por diversas sentencias del Tribunal General, ahora se alza la tesis del Tribunal de Justicia aclarando que también en la Unión Europea el principio de la libertad de expresión se debe tener en cuenta a la hora de analizar la prohibición de registro de marcas contrarias a las buenas costumbres. Esta afirmación puede llevar a una aplicación menos estricta de la prohibición que, incluso, puede ser trasladable a otros tipos de marcas, especialmente a aquellas que por su naturaleza comunicativa podrían ampararse en el principio de libertad de expresión comercial, como por ejemplo, como ya dijimos, ya ocurre con la publicidad. 